\title{
On the Impact of Satellite Communications over Mobile Networks: An Experimental Analysis
}

\author{
Engin Zeydan, Member, IEEE, Yekta Turk, Member, IEEE,
}

\begin{abstract}
Future telecommunication systems are expected to co-exist with different backhauling nodes such as terrestrial or satellite systems. Satellite connectivity can add flexibility to backhauling networks and provide an alternative route for transmission. This paper presents experimental comparisons of satellite and terrestrial based backhaul networks and evaluates their performances in terms of different Key Performance Indicators (KPIs) including Channel Quality Index (CQI), Modulation Coding Scheme (MCS) index, Downlink (DL) throughput, Frame Usage (FU) ratio and number of Resource Block (RB) utilization. Our experimental satellite network system uses a real satellitebased backhaul deployment and works in Ka band. As a benchmark, we compare our system with terrestrial network with regular cellular backhaul connection. Our experiments reveal three main observations: The first observation is that problems with FU ratio and number of $R B$ utilization exist in satellite eNodeB even though a single test user equipment (UE) with high CQI and MCS index values is connected. The second observation is that in satellite link relatively low numbers of Protocol Data Units (PDUs) are generated at Radio Link Controller (RLC) layer compared to the Packet Data Convergence Control (PDCP) layer. Finally, our third observation concludes that the excessive existence of PDCP PDUs can be due to utilization of General Packet Radio Service (GPRS) Tunneling Protocol-User Plane (GTP-U) accelerator where an optimal balance between the caching size and the number of UEs using satellite eNodeB is needed. Hence, the existence of a trade-off between the supported number of UEs using satellite link and the GTP-U acceleration rate is also revealed with our experimental results.
\end{abstract}

Index Terms-LTE, satellite communications, mobile network, field experiments.

\section{INTRODUCTION}

Long Term Evolution Advanced (LTE-A) is already putting a heavy burden on backhaul networks and various advanced techniques are proposed to improve microwave backhaul links including Adaptive Modulation and Coding (AMC), interference mitigation/cancellation techniques, higher order modulations, packet header compression, frequency diversity and multiple-input multiple-output (MIMO). This bottleneck problem of backhaul links gains more momentum as number of small cells inside Mobile Network Operators (MNOs)' network infrastructure is starting to soar. To carry this enormous amount of data traffic generated inside cells by end-users and Internet of Things (IoT) devices, in addition to Radio Access Network (RAN) level enhancements backhaul links have to be redesigned as well. Otherwise, backhaul links will soon be

E. Zeydan is with Centre Technologic de Telecomunicacions de Catalunya, Castelldefels, Barcelona, Spain, 08860. Email: engin.zeydan@cttc.cat

Y. Turk is a Mobile Network Architect based in Istanbul, Turkey. 34746. E-mail: yektaturk@ieee.org the bottleneck that will put whole proper operations of end-toend system into trouble. For these reasons, designing backhaul links is the next critical issue for Fifth Generation (5G) networks. For backhaul connection, various communication mediums can be considered as candidates such as microwave radio, copper Digital subscriber line (DSL), optical fiber, millimeter wave (mmWave) or satellite. The choice may depend on many factors including cost, performance, bandwidth demand, capacity and often many more. In particular, satellite point-to-point links that can exploit the benefits of satellite networks can be considered for reliable backhauling without interference to other cells or access links. Hence, satellites can play a key role for reliable service delivery in $5 \mathrm{G}$ networks and are already included in several 5G use cases [1].

On the other hand, providing ubiquitous coverage is one of the major requirements that needs to be satisfied within $5 \mathrm{G}$ networks. Next generation 5G wireless solutions are expected to embrace both satellite and cellular solutions [2], [3]. Due to cost per bit reductions of current state-of-the-art satellite technologies, MNOs have started to select satellite technology as a promising backhaul solution inside their cellular infrastructure. In fact, satellite networks can provide MNOs the opportunity to extend their coverage range in rural or remote areas of the country where existing infrastructure is not available (due to challenging topology of the geography) or limited in terms of network capacity. Hence, providing backhaul using satellite links in cellular networks can be a practical solution to provide low cost and timely delivery of connectivity services for hard to serve areas (such as mountains, islands, etc). For example, seamless integration and convergence with 5G terrestrial systems can support various vertical use cases and drive growth in sectors such as backhaul and trunking, transportation (aero, land, maritime) with mobile communications, media and entertainment with broadband services and public safety during disaster relief and emergency response situations.

Satellites can also complement next generation 5G terrestrial systems and provide substantial economic and societal benefits. For example, MNOs can have the option of not building a new cellular backhaul infrastructure in rural or remote areas depending on return-of-investment over infrastructure. The usage of satellite network for backhaul can reduce the infrastructure investment cost while providing coverage to large-geographical areas of the country.

In light of these, it is inevitable that satellite networks will integrate with other networks including $5 \mathrm{G}$ cellular networks. Support for satellite communications is considered to be an essential capability of the 5G technology. Moreover, satellites 
can be used to support key usage scenarios of 5G including enhanced Mobile Broadband (eMBB), massive Machine Type Communications (mMTC) and Ultra-reliable low latency communications (URLLC). For example, satellites can be used to carry high bandwidth High Definition (HD) content via High Throughput Satellites (HTSs) in Geosynchronous (GEO), Medium Earth Orbit (MEO) and Low Earth Orbit (LEO) in eMBB scenarios, can scale to support future IoT communications in mMTC scenarios and can play a role in low latency by delivering the same content to mobile base stations (BSs) or multicasting the content to caches of individual cells in URLLC scenarios. For URLLC applications, GSMA Intelligence report in [4] states that the content for services that require less than $1 \mathrm{msec}$ delay time should have all content served from a physical location that are very close to user equipment (UE), possibly at the base of every cell. In fact, applications such as video on demand streaming, virtual/augmented reality or tactile Internet necessitate the requirement to move network capacities and capabilities to the edge [5]. Hence when the transition to 5G occurs, these new contents that require low latency need to be moved to the edge which will also require many new locations. In these situations, satellites can help 5G networks to meet their sub$1 \mathrm{~ms}$ latency requirements by delivering commonly accessed content to mobile BSs. This is even possible even if no fiber connection at the dedicated site is available. According to Electronics Communication Committee (ECC) report 280 approved on April 2018 [6], satellite can play an important role to connect and update large number of edge servers that next generation mobile networks will require. Therefore, by multicasting content to caches that are located at individual cells, satellite multicast can become a viable option when Content Delivery Networks (CDNs) become ultimately densified. However, the success of satellites also depends on their capabilities to provide cost/bit reduction compared to terrestrial systems as well as adequate throughput improvements to provide $5 \mathrm{G}$ services for backup and offloading purposes. Moreover, large delays experienced by satellite links are one of the significant impediments to use this technology in traditional cellular networks, either in Long Term Evolution (LTE) currently or $5 \mathrm{G}$ in future.

\section{Motivation AND RELATED Work}

Satellite communication can be carried out by four different satellite categories namely, High Earth Orbit (HEO), LEO, MEO and GEO satellites that are orbiting at different altitudes around the Earth. In general, GEO and MEO satellites are used for communication purposes, especially for satellite backhaul applications.

Several works exist in the literature that study satellite integration with cellular networks [7], [8], [9], [10], [11], [12], [13], [14]. Different satellite-5G integration use cases as well as the latest initiatives and challenges in future $5 \mathrm{G}$ terrestrial and satellite integration are summarized in [7]. In [7], the authors have also investigated the impact of impairments in a typical satellite channel in LTE waveform design as well as in L1 and L2 procedures. The paper in [8] uses a realtime simulator for satellite backhauling of moving Evolved
Node Bs (eNodeBs) where LTE network and satellite links are emulated. The authors in [15] are proposing a new radio resource management algorithm for multimedia content distribution over satellite networks.

The authors in [11] study the integration of satellite and terrestrial communication networks and validate such integration with a testbed including a satellite emulator for backhaul support. The paper in [12] studies key technical challenges (mostly related to PHY/MAC layers) as well as architectures for incorporation of satellites into $5 \mathrm{G}$ systems. The paper in [13] presents several enabling techniques to reuse the existing terrestrial air interface for transmission over satellite links. The paper in [14] proposes a Downlink (DL) scheduling strategy in an integrated terrestrial-satellite network to enhance spectrum efficiency, fairness and capacity. The authors in [16] have reviewed the benefits of integration of satellite and terrestrial links to provide a network with wireless backhaul. The paper in [17] identifies the technical challenges associated with the convergence of satellite and terrestrial networks to provide quality-of-service (QoS) similar to high bandwidth terrestrial networks for end-users. Performance evaluation and research challenges of multi-satellite relay systems with cooperative transmission in Time Division Multiple Access (TDMA)-based architecture are given in [18]. For delivering TV services, Rajeev Kumar et al. in [19] investigate the usage of different wireless links (including satellite, $\mathrm{WiFi}$, and LTE/5G mmWave) to improve TV distribution penetrations.

Standardization bodies including 3rd Generation Partnership Project (3GPP) [20], European Telecommunications Standards Institute (ETSI) [21], International Telecommunication Union (ITU) [22] as well as joint initiatives such as 5G Infrastructure Public Private Partnership (5G PPP) [23] have also considered satellite networks in conjunction with terrestrial communication systems. For instance, 3GPP Release 15 has recently drafted a technical study on New Radio (NR) to support Non-Terrestrial Networks (NTNs) [20]. European Commission (EC) funded 5G PPP projects have also been launched within H2020 framework that focus on satellite communication. SANSA (Shared Access terrestrial-satellite backhaul Network enabled by Smart Antennas) project* investigates the utilization of extended $\mathrm{Ka}$ band for backhaul operations to improve spectrum efficiency. SaT5G (Satellite and Terrestrial Network for $5 \mathrm{G})^{\dagger}$ project focuses on plug-andplay integration of satellite communications into $5 \mathrm{G}$ network for eMBB use case. ESA ARTES SATis5 (Demonstrator for Satellite-Terrestrial Integration in $5 \mathrm{G}$ Context $)^{\ddagger}$ project builds a large Proof-of-Concept (PoC) testbed to enable satelliteterrestrial convergence into $5 \mathrm{G}$ context focusing on eMBB and mMTC scenarios.

Additionally, various works investigate satellite communication in Ka band [9], [10], [24], [25]. The authors in [9] focus on extending the LTE broadband service using a megaconstellation of LEO satellites that are deployed in Ka-band. An Uplink (UL) signal-to-interference-plus-noise ratio (SINR)

\footnotetext{
*https://sansa-h2020.eu/

${ }^{\dagger}$ http://sat5g-project.eu/

${ }^{\ddagger}$ https://artes.esa.int/projects/satis5
} 


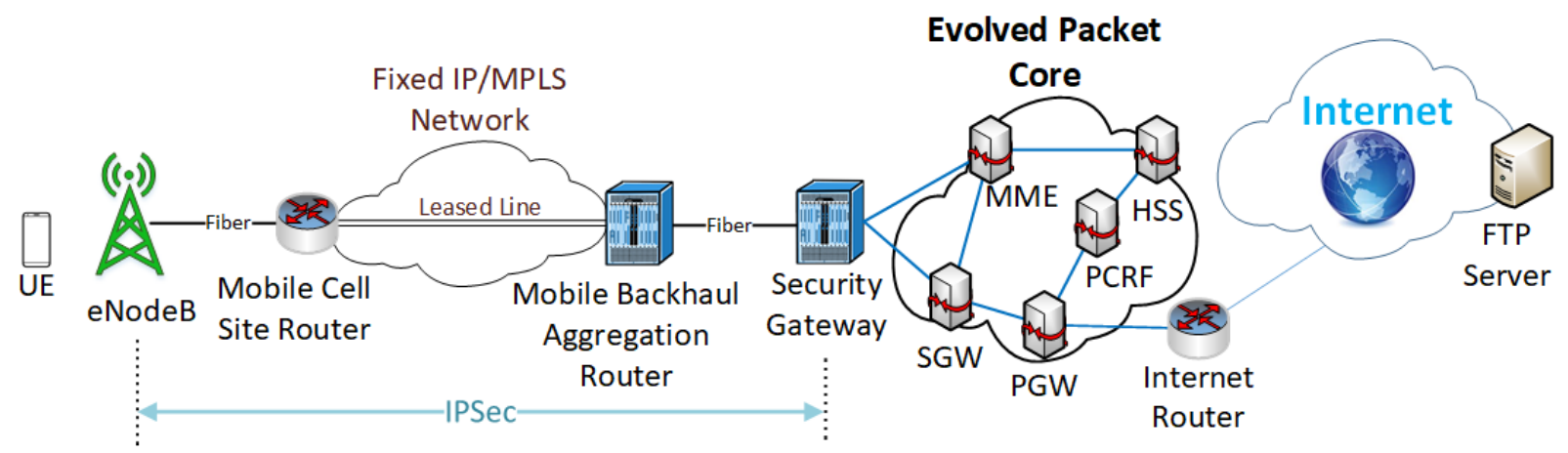

Fig. 1: General architecture of the terrestrial network.

probabilistic model for Ka band in HTSs is studied in [10]. The authors in [24] focus on beam tracking methodology in Ka-band for UAV-satellite communication systems. The paper in [25] provides an overview of advancements in many satellite based communication systems utilizing $\mathrm{Ka}$ band frequency band.

In operational networks, to compensate against the excessive latency existent in satellite links, GPRS Tunneling Protocol (GTP)-User Plane (GTP-U) accelerator is considered to be a viable solution by satellite modem vendors [26], [27]. The patent in [28] has applied acceleration function to GTP Protocol Data Units (PDUs) for traffic flows over satellite links via utilization of caching, pre-fetching and web acceleration methods. GTP-U accelerator is mainly useful for satellite links to mitigate the effect of long propagation delays that signal experiences.

Although most of the above papers have investigated cellular-satellite integration aspects, some limitations of the considered scenarios exist. For example, some related works either investigate performance over satellite emulators testbed implementations or work on virtualized environments of realtime simulators [8], [11]. These results lack relevant realistic requirements including the combined effect of LTE radio link with satellite-based backhaul connection to Evolved Packet Core (EPC). The aim of this work is to show how performance of satellite-based backhaul solution behaves in a real-time operational network and in comparison with regular terrestrial network. An important contribution of this paper is to complement literature works on integrated satellite-terrestrial networks by presenting an end-to-end complete and realistic validation results of the considered satellite-based backhaul system. Regarding that aspect, our proposed analysis works around the deployment of a satellite-based backhaul architecture, analysis over a wide range of key performance indicator (KPI) measurements including number of resource blocks (RBs) utilization, Frame Usages (FUs) ratios, Channel Quality Indicator (CQI), Modulation Coding Scheme (MCS) index, Packet Data Convergence Control (PDCP) throughput, number of PDUs in Radio Link Controller (RLC) and PDCP layers and MIMO Transport Block (TB) usage and comparisons with an operational terrestrial cellular network. More specifically, a eNodeB that connects a test UE to EPC via satellitebased backhaul is considered and the impact of satellite link latency on resource allocation schemes of satellite eNodeB is investigated.

Our experimental evaluations are conducted at minutes-level intervals which provide a fine grained characterization of the considered satellite-based backhaul deployment scenario. The analysis results reveal observed problems and trade-offs when KPIs of experimental satellite-based backhaul and terrestrial networks are compared. All these considerations have allowed us to understand the behaviour satellite-based backhaul architecture under real operating conditions. Our contributions in this paper can be summarized as follows:

- proposing a satellite-based backhaul architecture for cellular networks and performing a real-world experimentation over satellite link that utilizes Ka band,

- investigating satellite-based backhaul architecture's performance impacts on radio level KPIs and their comparisons with terrestrial cellular network's KPIs,

- revealing three distinct observations based on existence of FU ratio and RB utilization problems, excessive number of PDCP PDUs and the trade-off between the support for higher number of UEs and GTP-U acceleration rate using the satellite-based backhaul link.

The structure of the paper is as follows: Section III presents system model, concepts and the proposed architecture. Section IV presents the experimental analysis results using KPI measurements from both satellite and terrestrial eNodeBs for comparison purposes. The paper ends with conclusions in Section V.

\section{SyStem Model AND ARCHITECTURE}

Fig. 1 shows end-to-end system architecture of the utilized testbed for the terrestrial network site. This traditional mobile network consists of RAN, transport and core networks. In LTE, the access network is called Evolved Universal Terrestrial Radio Access Network (E-UTRAN) and the core network is EPC with all Internet Protocol (IP)-based connection. E-UTRAN is composed of eNodeBs, UEs whereas EPC includes Packet Data Gateway (P-GW), Serving Gateway (S-GW), Policy and Charging Rules Function (PCRF), Home Subscriber Station (HSS) and Mobility Management Entity (MME). 


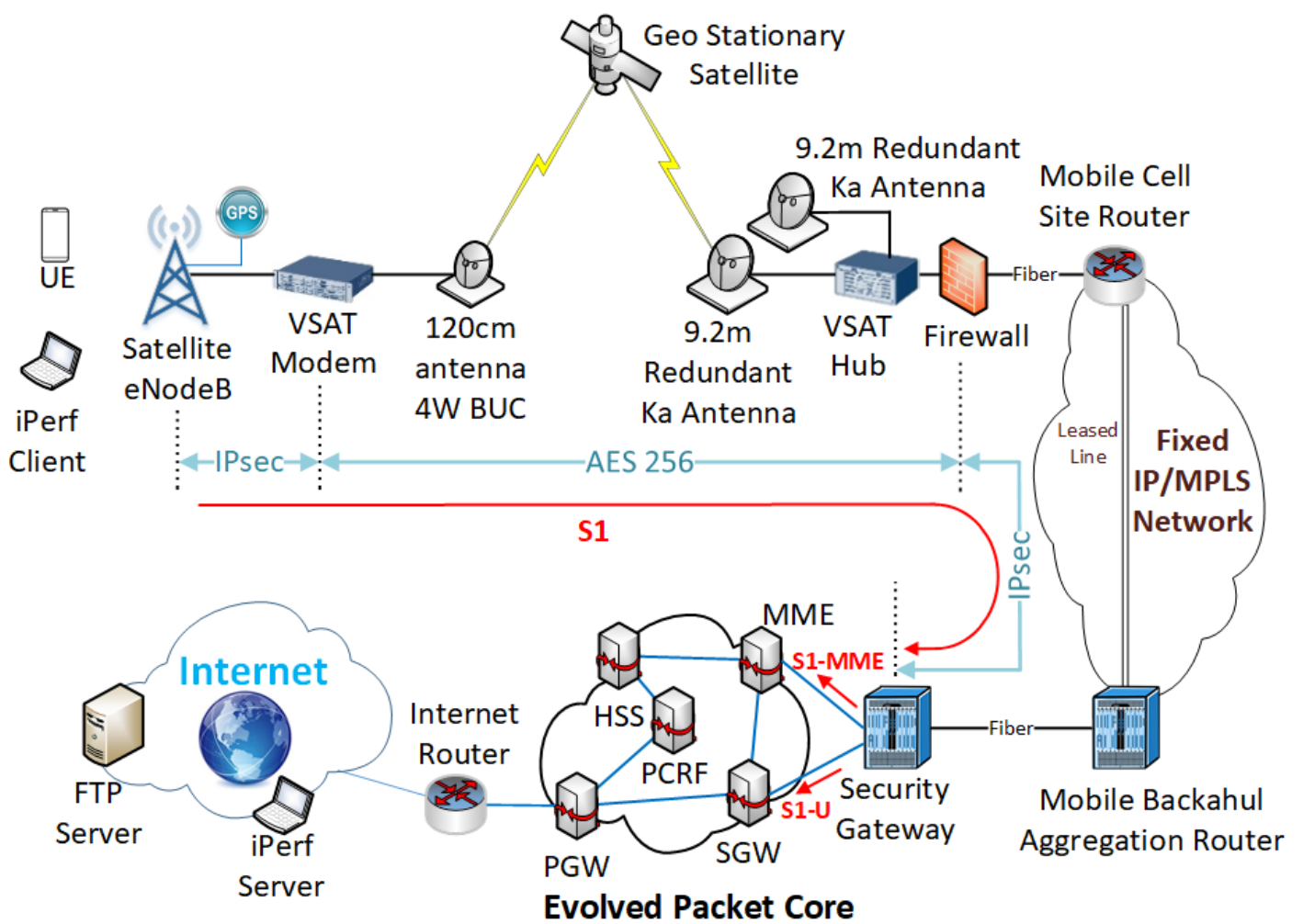

Fig. 2: General architecture of the satellite-based backhaul network.

A. General architecture of the satellite-based backhaul network

Fig. 2 represents a high-level architecture and key components of a GEO satellite-based backhaul for mobile network communication. We consider a GEO satellite that can provide satellite service to mobile network. In this setup, S1 interface between eNodeb and EPC is transported via satellite. LTE supports satellite transmission over S1 interface. Very Small Aperture Terminal (VSAT) modem is connected to the eNodeB with an Ethernet cable. VSAT hub and modem communicate over GEO satellite. VSAT hub and VSAT modem perform optimization to the $\mathrm{S} 1$ traffic as well as modulation of signal to be transmitted over the satellite link. In addition, VSAT modem is capable of both Internet Protocol Security (IPsec) setup and GTP-U acceleration. A $120 \mathrm{~cm}$ antenna is deployed on the site for satellite access. Two redundant $9.2 \mathrm{~m} \mathrm{Ka}$ antennas, one VSAT hub and one firewall are located in ground station. VSAT hub is connected to firewall via Ethernet. Between VSAT modem and firewall, Advanced Encryption Standard (AES)-256 encryption is used to encrypt traffic since IPsec usage is not feasible over satellite networks. The firewall is used for encryption of the satellite traffic and establishment of a new IPsec tunnel with the Security Gateway (SecGW) in the mobile core network. In MNOs' networks, Cell Site Routers (CSRs) are installed at eNodeB sites to transmit traffic to the IP/Multiprotocol Label Switching (MPLS) backhaul network. However due to this topology where eNodeB S1 traffic is transmitted over satellite, mobile CSR is installed on the ground station where VSAT hub is located. The CSR is connected to a mobile Mobile Backhaul Aggregation Router (MBAR) via a leased line over a carrier of the fixed network operator. Links from many CSRs are collected in the MBAR and forwarded to the LTE SecGW. The purpose of the SecGW is to terminate IPsec tunnel set up and (d)encrypt S1 traffic. SecGW is connected to EPC and EPC is connected to the Internet via a high-capacity Internet router as given in Fig. 2. GTP-U acceleration method is applied mutually at both VSAT modem and VSAT hub [26].

\section{B. Ka band utilization for satellite-based backhaul}

HTSs are characterized by many small beams with high gains that also allow multiple frequency re-use. It can provide high capacity communication with reduced costs [29]. Ka band frequency utilization is getting common in HTSs [25]. In fact, classical frequency bands such as $\mathrm{C}$ and $\mathrm{Ku}$-band are getting congested due to increasing number of satellite communication systems. For this reason, utilization of $\mathrm{Ka}$ bands can provide higher bandwidths compared to L-/S- bands and is more suitable for broadband services due to high frequency usage. Moreover, higher frequencies avoid the interference with terrestrial communication systems, enable the reduction of system components both in ground and space segments and enable higher antenna gains and directivity. However, as frequency increases small perturbations on the atmosphere (e.g. rain attenuation) can have high impact on link quality of the propagating waves. This affects end-users' experienced QoS. For this reason, it is critical to observe the effect of $\mathrm{Ka}$ 


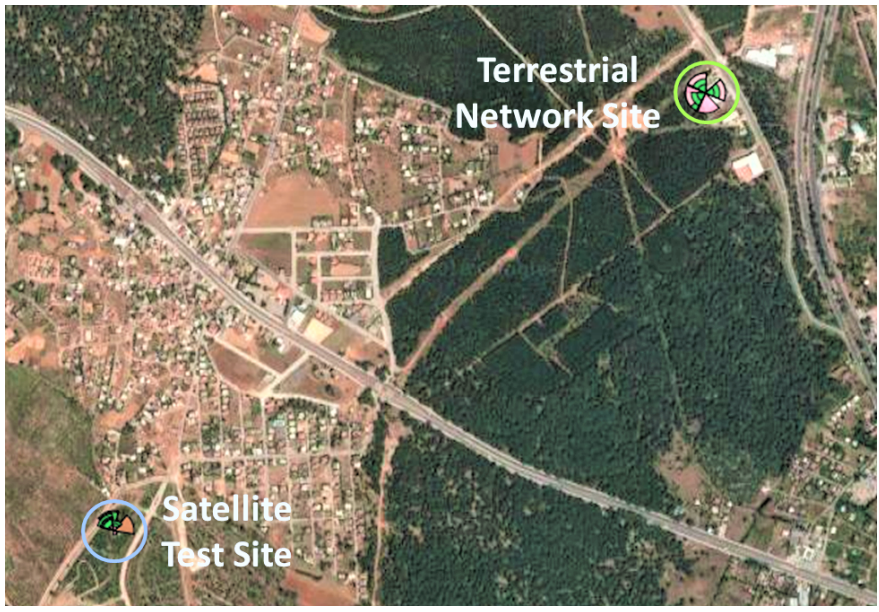

Fig. 3: Experimental test site locations of terrestrial and satellite networks.

band utilization over satellite links with experimental trials and compare various KPIs with legacy terrestrial networks.

\section{EXPERIMENTAL RESULTS}

In this section, our focus is to obtain empirical assessments of the satellite-based backhaul set-up and compare it with a terrestrial $4 \mathrm{G}$ cellular mobile radio communication network using different KPIs. Observations of different KPIs can yield insights into identifying trade-offs under actual network deployment conditions. For this reason, a real-time prototype of satellite-based backhaul is deployed and tested over the infrastructure of the operator.

In the presented work, the deployed satellite-based backhaul system constitutes a single satellite eNodeB where a single test UE is connected to an EPC for end-to-end system implementation. Fig. 3 shows experimental test site locations of the utilized terrestrial and satellite networks. The KPI performance differences between a satellite and terrestrialbased backhaul mobile system enable us to evaluate their comparative performances and observe any potentially existing trade-offs.

\section{A. Satellite components and configurations}

Our satellite system is HTS and uses Ka band frequencies between $19.828 .662 \mathrm{kHz}-19.891 .412 \mathrm{kHz}$ for DL and $29.590 .047 \mathrm{kHz}-29.594 .041 \mathrm{kHz}$ for UL. Therefore, spectrum bandwidths are $70 \mathrm{Mhz}$ and $4 \mathrm{Mhz}$ for DL and UL respectively.

The DL transmission is between the satellite and the eNodeB whereas UL is vice-versa. Usually, dedicated bandwidth for DL is higher than UL. The total bandwidth is the summation of UL and DL bandwidths. In this paper, we utilize a GEO stationary satellite which is $35.786 \mathrm{~km}$ away from Earth. Considering the GEO satellite altitude and its position in an equatorial plane, the distance between the GEO satellite and a point on the Earth's surface can be ranged between 35.786 $\mathrm{km}$ (if the point is on the Equator) and about $40.000 \mathrm{~km}$ (near to one of the pole). As a consequence, one-way propagation

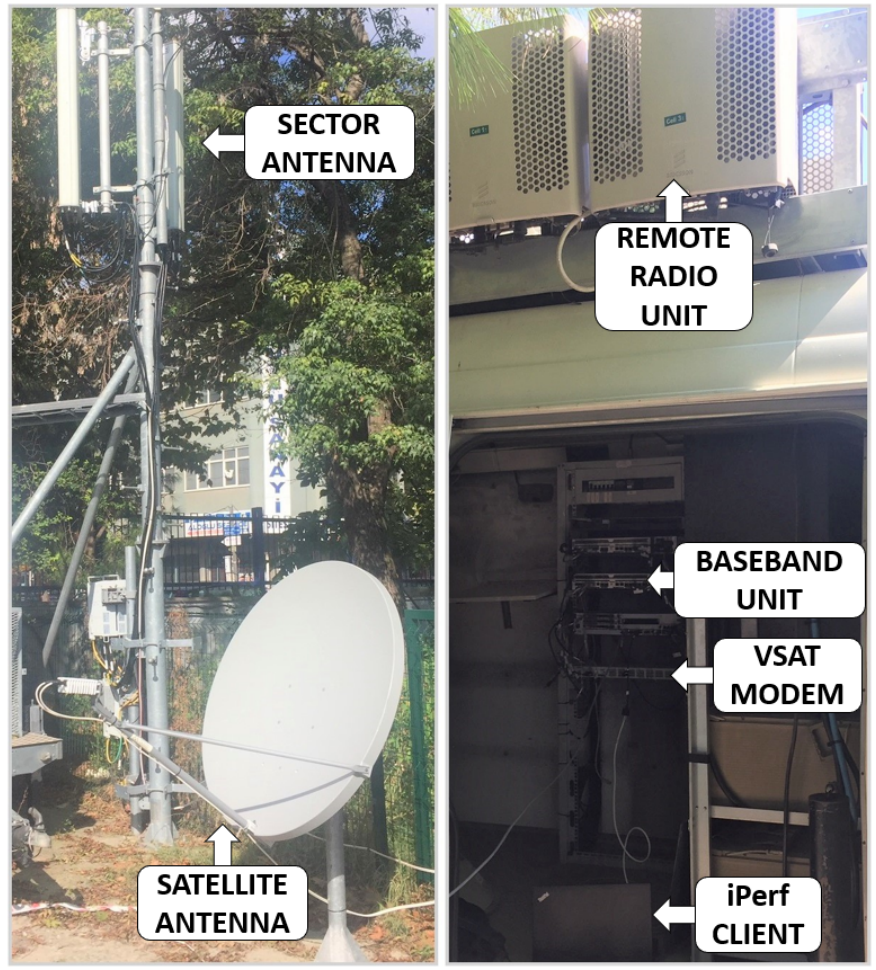

Fig. 4: Installed equipment and connections on the satellite test site. Left: Satellite and sector antennas. Right: RRU, BBU, VSAT modem and Iperf client.

delay of GEO satellite transmission can be calculated to range between

$$
\frac{35,786 \mathrm{~km}}{3 e 5 \mathrm{~km} / \mathrm{s}}=119 \mathrm{~ms} \leq T=\frac{d}{c} \leq \frac{40,000 \mathrm{~km}}{3 e 5 \mathrm{~km} / \mathrm{s}}=133 \mathrm{~ms} .
$$

Hence, the communication delay between two ground stations through the satellite is $238 \mathrm{~ms} \leq 2 T \leq 266 \mathrm{~ms}$.

\section{B. Testbed network functions}

For experimental end-to-end tests over the considered networks, we used a notebook with SIM card as an iPerf [30] client and a test UE with TEMS [31] tool installed to collect radio-level KPIs. A File Transfer Protocol (FTP) server that is located on the Internet is used to download a file via UE. Same tests were conducted both in terrestrial and satellite test sites. Iperf tool is used to generate a User Datagram Protocol (UDP) flow between the iPerf client and the iPerf server. The principle objective of the experimental test system is to allow realistic evaluations of satellite-based backhaul deployment in a real-operator environment. Fig. 4 shows different types of network devices that are used during experimental trials. These components are:

1) Satellite antenna: The antenna is fixed to the ground to combat against environmental effects after Line-of-Sight (LOS) is adjusted towards the satellite.

2) Sector antenna: A sector antenna is a directional antenna for outdoor environment that provides high gains. Sector 


\begin{tabular}{|c|c|c|}
\hline $\begin{array}{c}\text { Measured } \\
\text { Protocol }\end{array}$ & $\begin{array}{c}\text { Upload Test } \\
\text { Completion Time(s) }\end{array}$ & $\begin{array}{c}\text { Download Test } \\
\text { Completion Time(s) }\end{array}$ \\
\hline TCP & 11.35 & 11.13 \\
\hline UDP & 10.98 & 10.89 \\
\hline
\end{tabular}

TABLE I: Test completion results of iPerf measurements taken from the satellite eNodeB.

antennas consist of an array of dipoles. The performance of these antennas depend on the size and shape of the reflector.

3) Baseband Unit (BBU): A BBU is a unit that processes baseband and is connected to Remote Radio Unit (RRU) via optical fiber.

4) RRU: A RRU performs radio frequency (RF) DL and UL channel processing. RRU communicates with BBU via a physical link and with wireless mobile devices via air interface.

5) VSAT Modem: An equipment that performs GTP-U acceleration and selected security protocols (e.g. IPsec, AES-256). It is also compatible to operate with LTE. It is connected to the satellite antenna and eNodeB.

6) iPerf Client: iPerf works in a client/server mode. A client sends data to the server for testing purposes.

Additionally, the deployment complies with the relevant features of LTE standards.

Table I presents iPerf measurements' delay responses for UL and DL from/to the satellite eNodeB. Bandwidth setting for iPerf test is limited to $50 \mathrm{Mbit} / \mathrm{s}$ to transfer approximately 60 MBytes of data. The results indicate that UDP traffic has low completion time compared to Transport Control Protocol (TCP) traffic. In IPerf tests, it is seen that tests with TCP were completed in both UL and DL by approximately 3 percent later than UDP. This demonstrates how TCP is affecting the applications used by test UE connected to satellite eNodeB. Differences between UL and DL test completion duration are caused by hardware differences between iPerf server and client. It is also related to the high number of simultaneous tests performed by the server.

In rest of the paper, Probability Distribution Function (PDF) of a KPI represents the percentage of the specific KPI's measurements at a specific value whereas Cumulative Distribution Function (CDF) represents the percentage of that specific KPI's measurements that are at least as good as a specific value.

\section{Performance in Downlink}

MCS and CQI values: Top subfigures of Fig. 5 marked with dark blue colors represent the average CQI values for both terrestrial and satellite eNodeBs. We can observe that satellite eNodeBs' link quality is high and a mean CQI value of 14.96 is observed during the observation time interval. This indicates good RF medium conditions in satellite-based backhaul network and there is no problem with RF signal quality for satellite test UE. On the other hand, terrestrial network's CQI values fluctuate and radio conditions are slightly poor compared to satellite eNodeB with an observed mean CQI value of 9.92. Red colored marks in third row of Fig. 5a and
Fig. 5b show MCS index values over the observation duration for terrestrial and satellite eNodeBs respectively. MCS index values in terrestrial eNodeB are fluctuating between 0 and 25 . This again validates that radio conditions are variable and worse than satellite network. In comparison, satellite network MCS index values are almost constant at maximum MCS index value of 25 . This indicates usage of high modulation scheme in satellite eNodeB with very small drop-offs at certain time intervals in DL. Note that there are many real-UEs connected to terrestrial eNodeB and only one test UE in satellite eNodeB. This is also another effect that can have a negative impact on observed average CQI and MCS index values in terrestrial eNodeB.

Number of RB utilization distributions: Fig. 6 shows number of RB utilization in DL for both terrestrial and satellite eNodeBs. From presented CDF and PDF plots, we can observe different distribution behaviours for number of RB utilization in terrestrial and satellite eNodeBs. For example, in Fig. 6 (a) $82 \%$ of number of RB utilization are at least as good as 40 RBs and in Fig. 6 (b) $87 \%$ of number of RB utilization are at least as good as 64 RBs. Similarly, Fig. 7 shows the boxplot for number of RBs utilization in DL for both terrestrial and satellite eNodeBs. The median values for number of utilized RBs for satellite and terrestrial eNodeB are 76.92 and 43.53 respectively. In terrestrial eNodeB, the variance of $\mathrm{RB}$ is observed to be narrower than satellite eNodeB. Although RF conditions seem relatively poor in terrestrial eNodeB, the scheduler utilizes RBs as many as possible where up to $50 \mathrm{RBs}$ are used for a given bandwidth of $10 \mathrm{Mhz}$. In comparison, in satellite eNodeB, RB utilization variance is larger.

FU ratio distributions: Fig. 8 shows FU ratios in DL for both terrestrial and satellite eNodeBs. CDF and PDF plots show the behaviour of FU ratios distribution. For example, in Fig. 8 (a) $55 \%$ of FU ratio measurements are at least as good as 68 and in Fig. 8 (b) $65 \%$ of FU ratio measurements are at least as good as 52. Similar observations and conclusions with DL RB utilization results can also be deducted from FU ratio observations of Fig. 8.

Fig. 5a and Fig. 5b (second rows marked with yellow colors) depict the FU ratios for both terrestrial and satellite eNodeBs respectively. Note that real UEs are connected to the terrestrial eNodeB during the observation period. The scheduling of UEs at each transmission time interval (TTI) (i.e. every 1 ms) may allow some UEs to have no resource allocations during this allocation interval. However as depicted in Fig. 5b, interestingly the same trend of DL FU percentage fluctuations are observed with satellite backhaul network as well. Note that in satellite eNodeB, FU percentage values are not expected to fall to zero values since only one test UE exists in the satellite network and additionally radio conditions are observed to be good (from observations of CQI and MCS index values as discussed previously) in satellite eNodeB.

For better visibility of the values in Fig. 5a and Fig. 5b, we also plot the boxplot for the FU ratio (\%) in DL for both terrestrial and satellite eNodeBs in Fig. 7. In this figure, median values of FU ratio for satellite and terrestrial eNodeBs are $60.66 \%$ and $66.87 \%$ respectively. Fig. 7 clearly validates a higher FU ratio in terrestrial eNodeB compared to satellite 


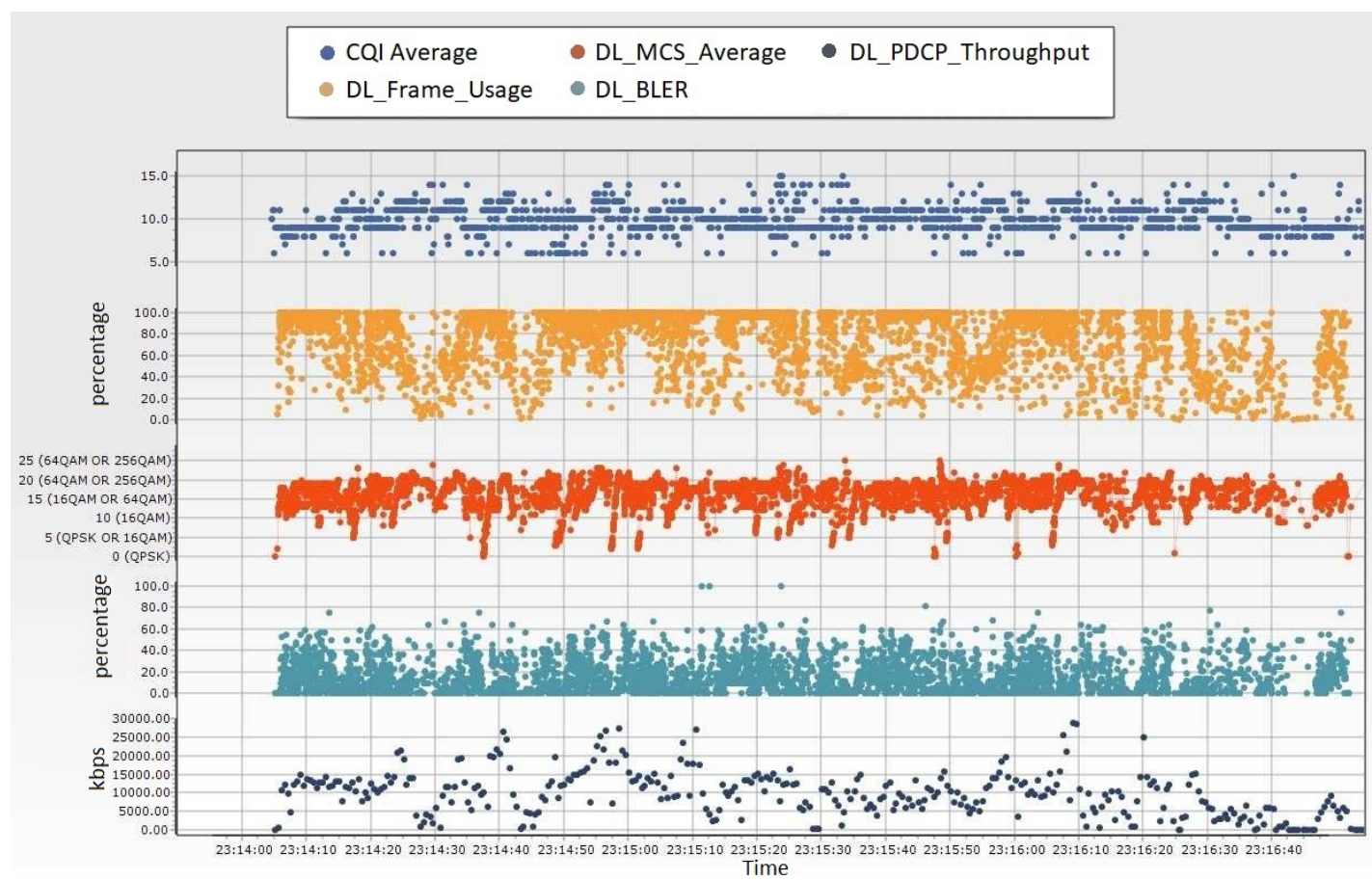

(a)

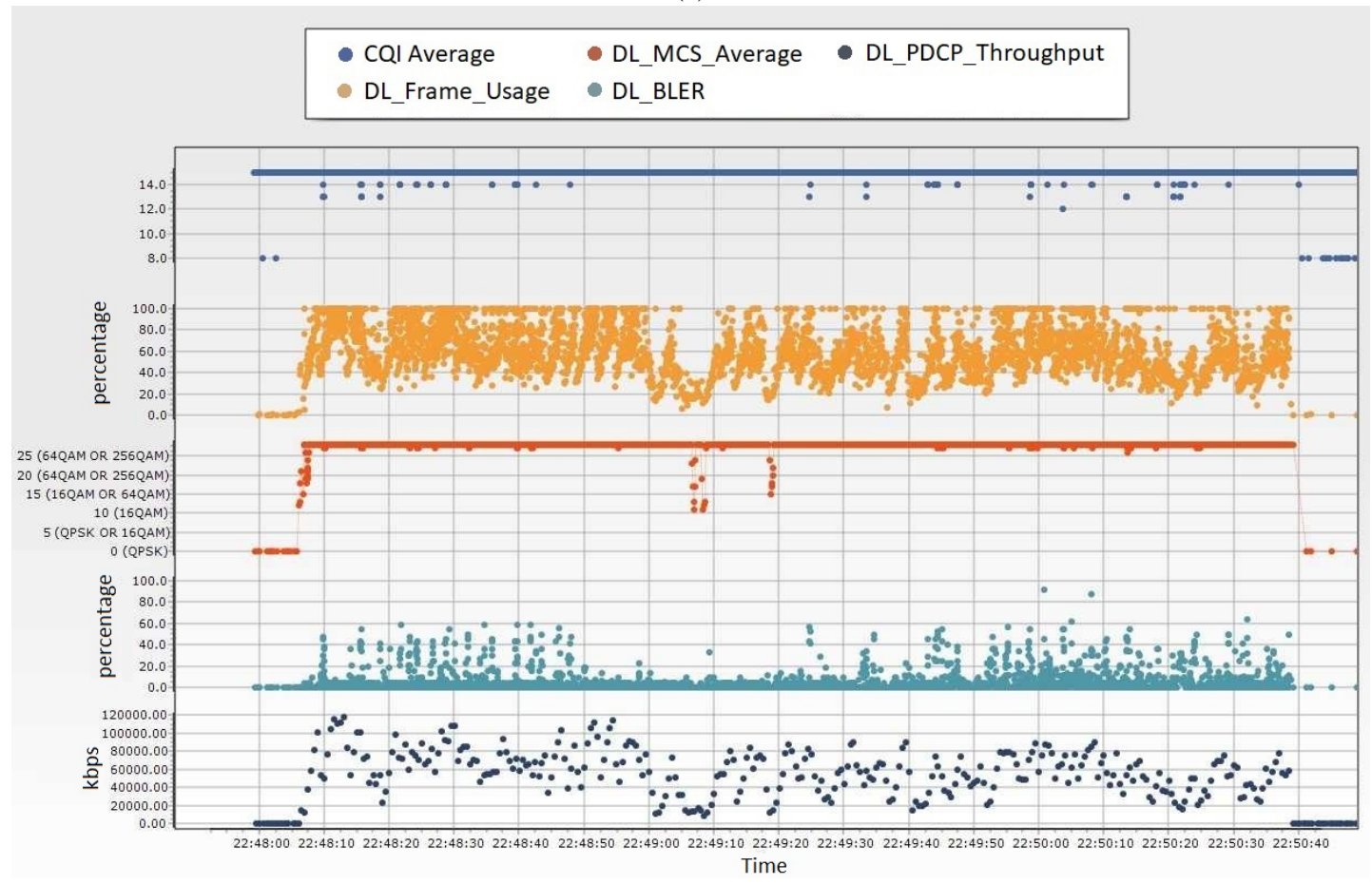

(b)

Fig. 5: Radio Performance KPIs in DL (a) Terrestrial eNodeB (b) Satellite eNodeB [Figures are best viewed in color.]

eNodeB. Similar to Fig. 6, satellite eNodeB cannot schedule the PDUs appropriately at Medium Access Control (MAC) layer due to existence of incomplete transmit buffer. This in turn reduces FU ratios as well as DL PDCP throughput even though only one test UE is connected to satellite eNodeB. An unexpected high variance on FU ratio for satellite eNodeB exists due to existence of unfilled transmit buffer and irregular PDU receptions caused by high delay and jitter in satellite link.
Block Error Rate (BLER) percentages: Light blue marked subfigures in fourth rows of Fig. 5a and Fig. 5b depict the DL BLER percentages for terrestrial and satellite eNodeBs respectively. Higher BLER percentages are observed in terrestrial eNodeB compared to satellite eNodeB. In general, when BLER percentage increases, MCS index drops as expected and subsequently throughput decreases. This has been observed in terrestrial eNodeB. Moreover, other UEs exist inside terrestrial 


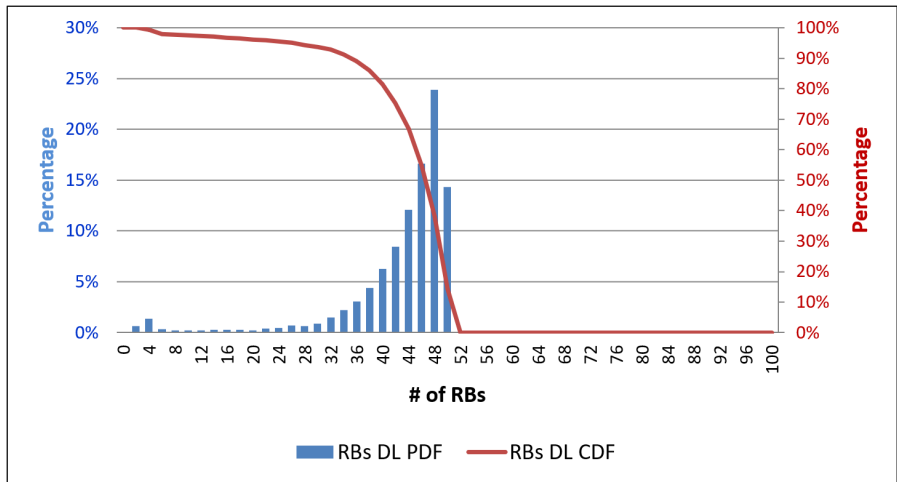

(a)

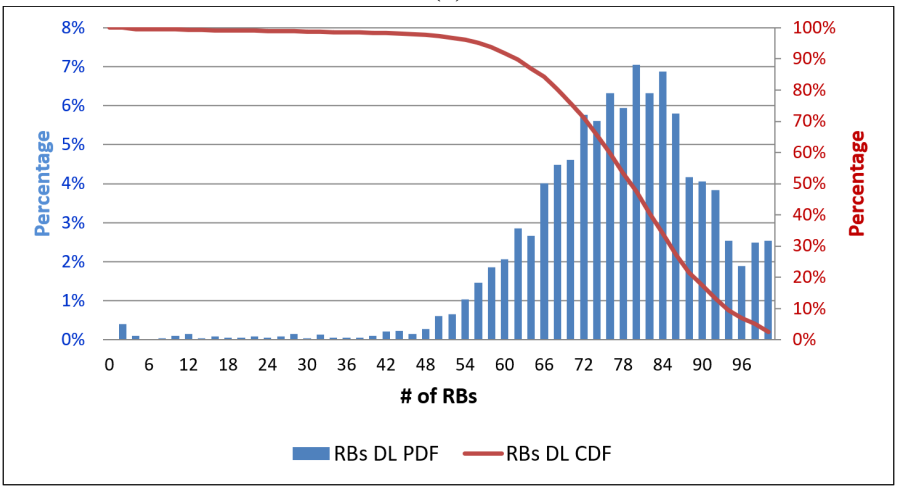

(b)

Fig. 6: PDF and CDF plots for DL number of RB utilization distributions in (a) Terrestrial eNodeB (b) Satellite eNodeB.

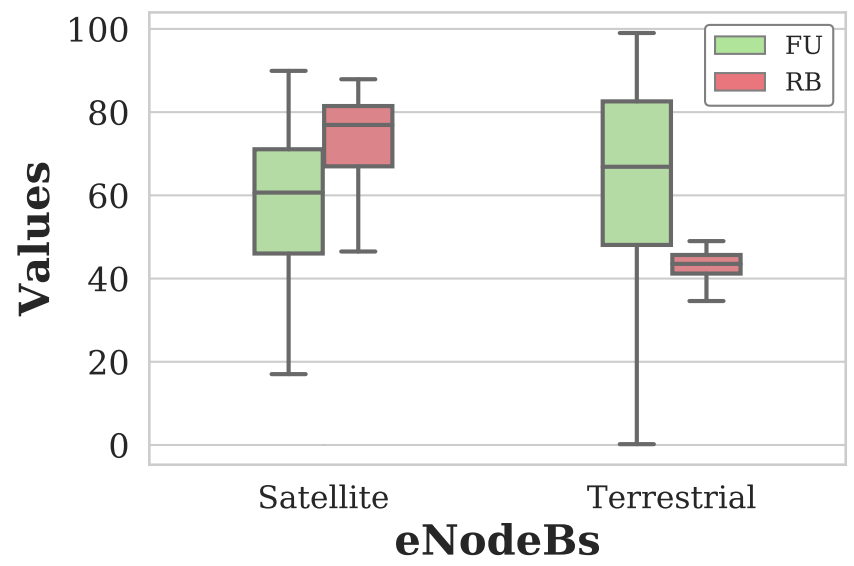

Fig. 7: Boxplot comparison values for number of RBs usage and the FU ratio (\%) in DL for terrestrial and satellite eNodeBs.

cell that contribute to higher BLER values. When satellite eNodeB results are analyzed, the error detections for PDUs that are sent by test UE are recognized very late by Hybrid automatic repeat request (HARQ) due to end-to-end latency in the network. This in turn has increased the BLER for satellite eNodeB.

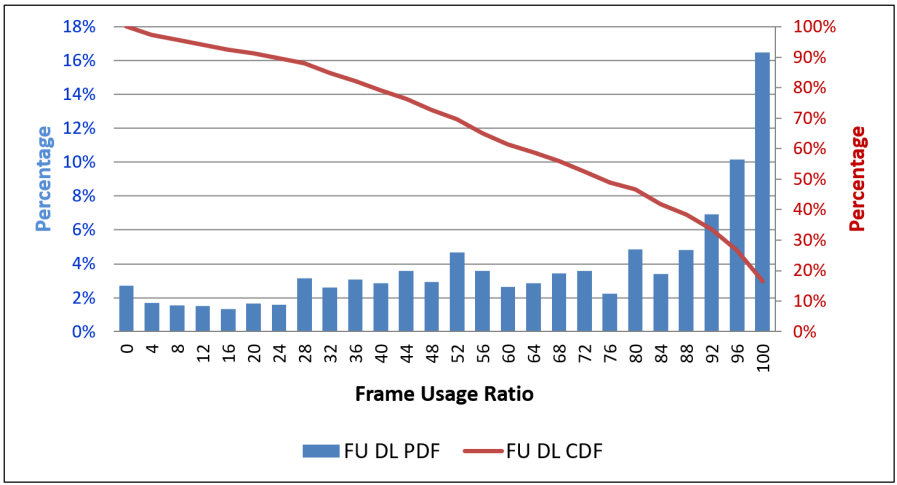

(a)

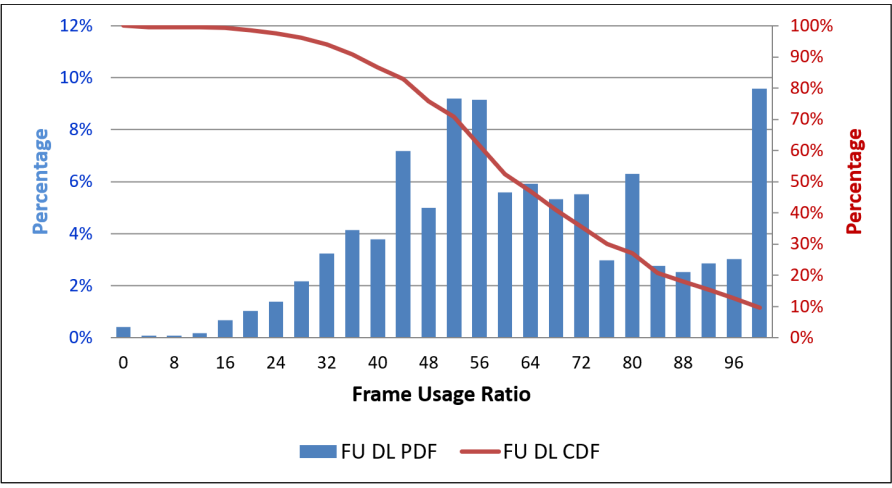

(b)

Fig. 8: PDF and CDF plots for DL FU ratio distributions in (a) Terrestrial eNodeB (b) Satellite eNodeB.

\section{KPI Relationship analysis}

In this section, we investigate the relationship between different LTE performance KPIs by varying one of them and observing its affect on the remaining KPIs. Monitored KPIs are DL throughput, CQI values, FU ratios, number of RBs utilization, BLER percentages, number of PDUs in RLC/PDCP layers, MCS index values and MIMO TB usage. In the following analysis, some metrics have been normalized to percentage values so that $100 \%$ corresponds to the maximum value. Hence, metrics with different ranges can be represented on the same graph. For instance, "RBs DL (in \%, normalized to 30 )" means that the maximum value of "RBs DL" in the graph is 30 . In that case, $50 \%$ would correspond to "RBs DL $=15$ ".

KPI relationship analysis based on number of $\mathrm{RBs}$ utilization in DL: Fig. 9 shows the average percentage values for CQI, MCS index and FU ratio on the right y-axis as well as average throughput values on left-y axis versus increasing number of RBs utilization on x-axis. Note that in Fig 9a and Fig 9b, CQI values are normalized with respect to maximum value of 10.5 and 15 and DL MCS index values are normalized with respect to maximum value of 16 and 28 respectively. Number of DL RBs is related to load in the cell. As number of RBs increases, throughput values of both satellite and terrestrial eNodeBs also increase accordingly as expected. For example in Fig. 9a together with $50 \mathrm{RBs}$ (for $10 \mathrm{Mhz}$ of bandwidth) around $18 \mathrm{Mbps}$ can be achieved, whereas in 


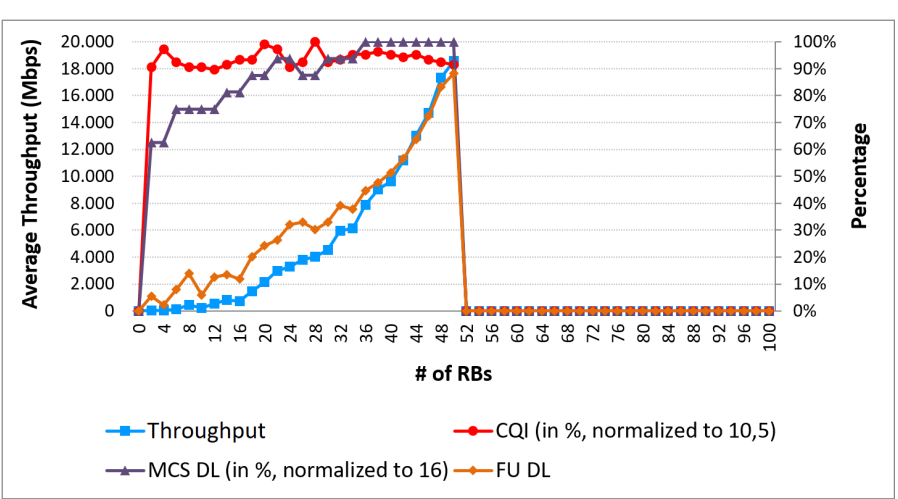

(a)

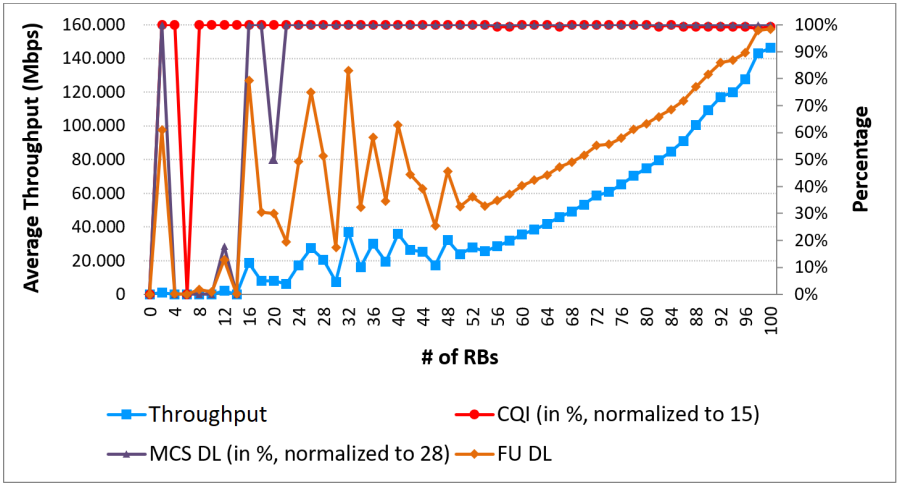

(b)

Fig. 9: All KPIs versus \# of RBs utilization relationship analysis for (a) Terrestrial eNodeB (b) Satellite eNodeB [Figures are best viewed in color]

Fig. $9 \mathrm{~b}$ the throughput raises above 140 Mbps with $100 \mathrm{RB}$ utilization (for $20 \mathrm{Mhz}$ of bandwidth).

Average MCS index and CQI values are at relatively maximum levels for satellite eNodeB in Fig. 9b. (Note that high fluctuations exist in Fig. 9b at the beginning for average MCS index values due to small number of averaged sample data at low number of RBs.) Due to RF conditions of terrestrial eNodeB, CQI and MCS index values fluctuate between $60 \%$ to $100 \%$ in Fig. 9a. Comparing DL FU ratio distributions when the number of RBs utilization increases in Fig. 9, we can observe that the increasing trend is slower in satellite eNodeB compared to terrestrial eNodeB. This is related to transmit buffering problem encountered in satellite eNodeB which is also discussed in more detail in subsequent sections.

KPI relationship analysis based on LTE DL FU ratio: Fig. 10 shows the relationship between FU ratio and other related KPIs. When the number of RB utilization percentages at a given $\mathrm{FU}$ ratio (in percentages) are compared between terrestrial and satellite eNodeBs in Fig. 10, a higher RB utilization percentage in terrestrial eNodeB is observed. For example at $32 \% \mathrm{FU}$ ratio, $\mathrm{RB}$ utilization percentage is around $70 \%$ for satellite eNodeB, whereas it reaches to $90 \%$ for terrestrial eNodeB. Fig. 10b shows that the number of utilized RBs can decrease as FU ratio increases. For instance, the number of RBs usage percentage is lower when FU ratio is 74 compared to the case when it is 72 . This result is in contrast

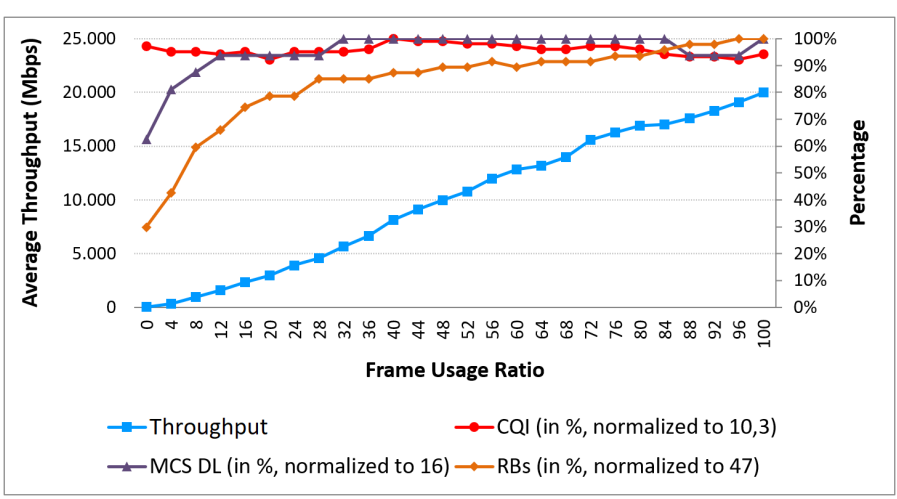

(a)

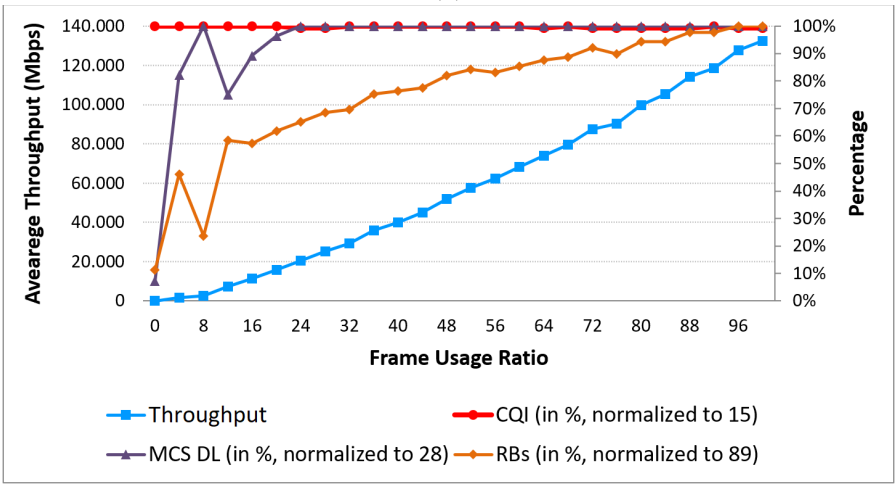

(b)

Fig. 10: All KPIs versus DL FU ratio relationship analysis for (a) Terrestrial eNodeB (b) Satellite eNodeB [Figures are best viewed in color]

with expectations and the observation results of terrestrial eNodeB in Fig. 10a. The main reason is again due to the transmit buffer not being filled up completely for appropriate scheduling of PDCP PDUs.

Fig. 10 also shows the effect of increase in MCS and its impact on PDCP throughput, FU and CQI. The effect of jitter and latency in satellite-based backhaul can be extracted from these KPIs of satellite eNodeBs. The transmit buffer is not full due to latency originated satellite backhaul link. For this reason, PDCP PDUs are kept inside transmit buffer until transmit buffer is full so that they can be scheduled later. The PDCP PDUs in the transmit buffer are scheduled to be transmitted quickly due to existence of high number of DL RBs. This results in empty TTI to be transmitted until new PDUs arrive which negatively effects the FU ratio (\%) over the satellite link. Due to existence of only one UE in satellite eNodeB and the impact of latency and jitter of the satellite link, the FU ratio has wide variance as also observed in Fig. 10b and Fig. 7. On the other hand, GTP-U accelerator yields satisfactory UE throughput in contrast to this large variance in FU ratio as also observed from Fig. 10b. Note that there is only one test UE inside satellite network and there is a tendency that DL FU ratio is expected to worsen in case the number of UEs increases.

DL PDCP throughput: Fig. 10 marked with purple color shows DL PDCP throughput values. As expected, DL PDCP 


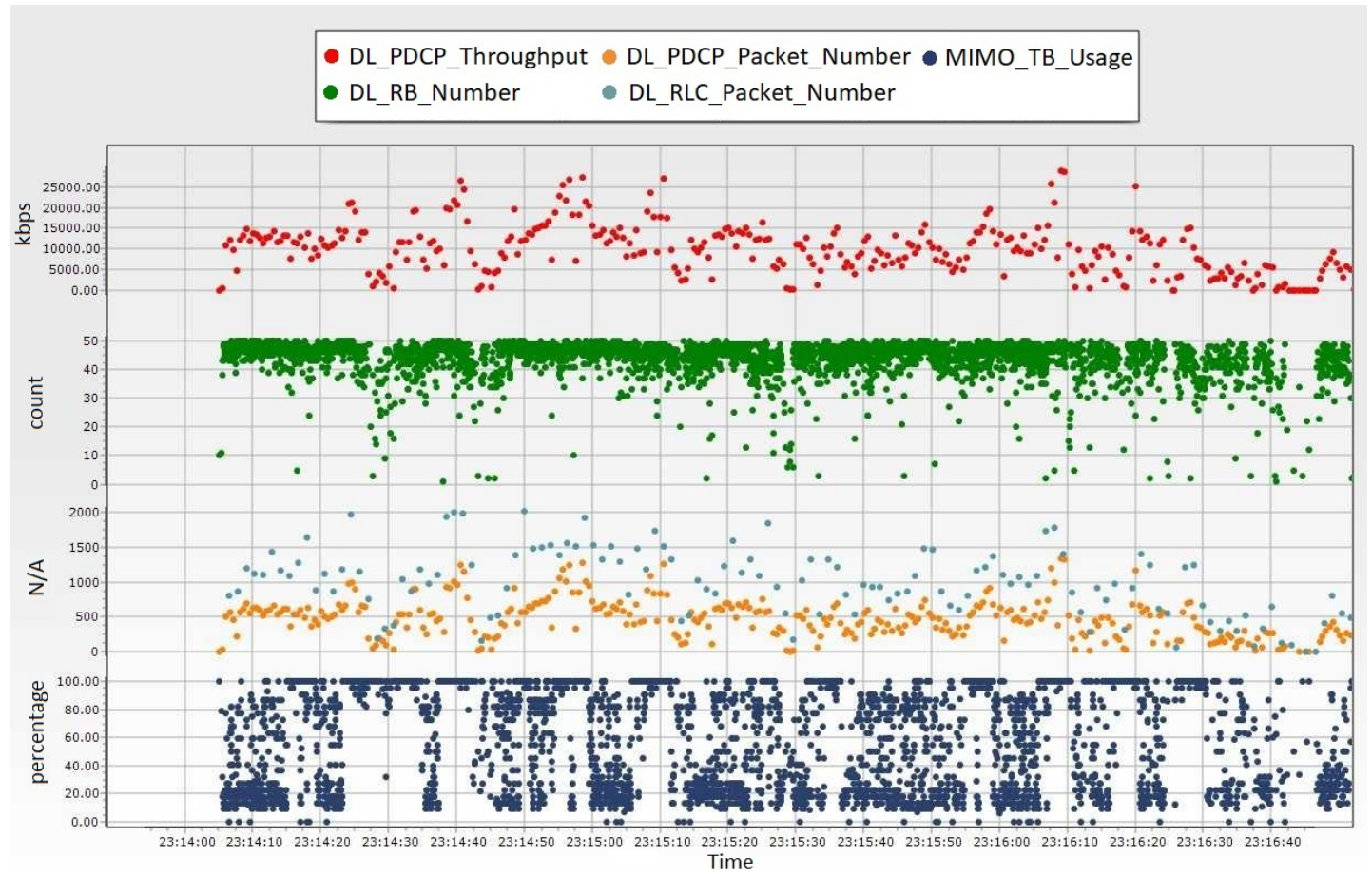

(a)

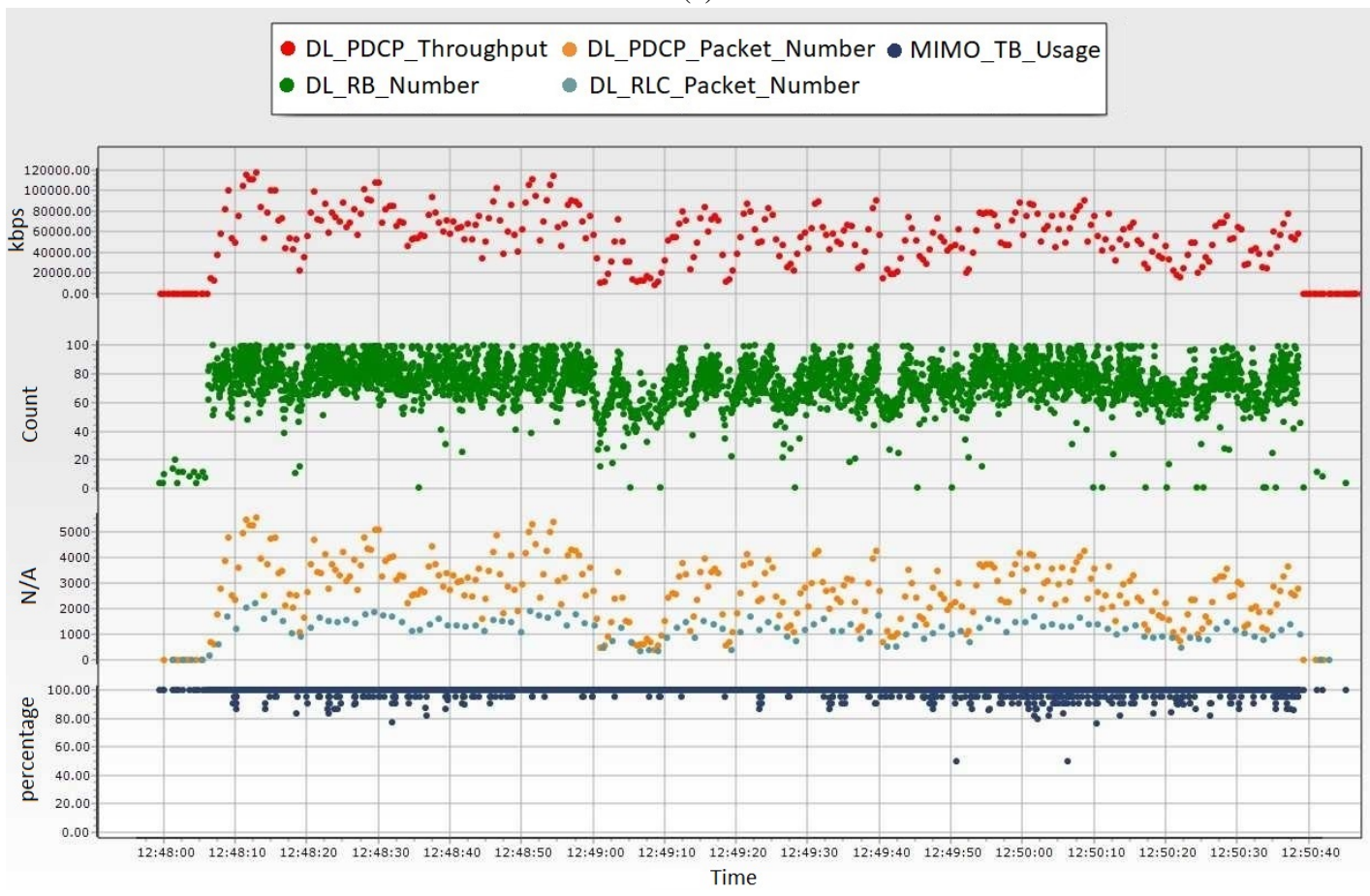

(b)

Fig. 11: Additional Radio Performance KPIs in DL for (a) Terrestrial eNodeB (b) Satellite eNodeB [Figures are best viewed in color.]

throughput values of both terrestrial and satellite eNodeBs increase as FU ratio increases in Fig. 10. At the same time, satellite eNodeB yields higher average DL PDCP throughput values compared to terrestrial eNodeB due to existence of single UE as shown in last row sub-figures marked with black colors of Fig. 5.

MIMO-TB usage percentage: Fig. 11a and Fig. 11b show
MIMO-TB usage percentage values which are marked in black color. In both figures, MIMO-TB values hit $100 \%$ which indicate that both terrestrial and satellite eNodeBs utilize MIMO. Due to existence of high number of UEs in terrestrial eNodeB, TB usage percentages have fluctuated over time. On the other hand, no significant TB usage percentage change occurs in satellite eNodeB, hence it is around $100 \%$. 


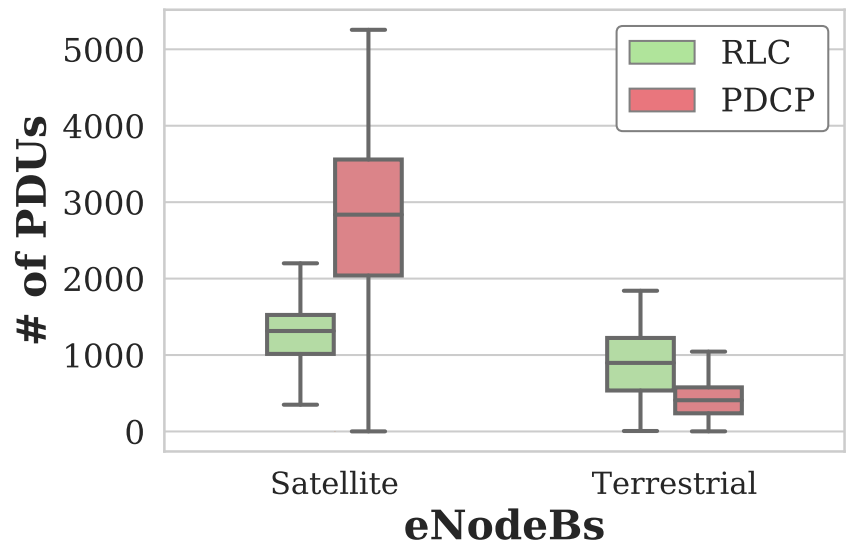

Fig. 12: Boxplot comparisons of number of PDUs at each radio protocol stack (RLC and PDCP) for both terrestrial and satellite eNodeBs.

Number of PDUs in PDCP and RLC layers: Light blue and yellow colors in third rows of Fig. 11a and Fig. 11b mark the number of PDUs in RLC and PDCP layers respectively. For more detailed analysis, Fig. 12 shows the boxplot of the number of PDUs in RLC and PDCP layers for both terrestrial and satellite eNodeBs over the same observation duration. We can observe that the median numbers of DL RLC PDUs are 897 for terrestrial eNodeB and 1314.5 for satellite eNodeB. On the other hand, median numbers of DL PDCP PDUs (that are related to PDCP throughput values) are 408.75 for terrestrial eNodeB and 2836.5 for satellite eNodeB. This is consistent with PDCP throughput values. The main observation that can be extracted from Fig. 12 is that the number PDUs in PDCP layer is higher than RLC layer in satellite eNodeB in comparison with terrestrial eNodeB. This is due to larger waiting period during transmit buffering stage in satellite eNodeB compared to terrestrial eNodeB. In satellite eNodeB, RLC layer aggregates more than one PDCP packets and sends them to lower layers as one RLC packet.

Main takeaways and observations: Our experimental results reveal three important observations in both terrestrial and satellite eNodeBs experiments using the KPI analysis.

The first observation states that FU ratio and RB utilization problems caused by the delay and jitter in the satellite link exist. The variances of FU ratio and number of RBs distributions are unexpectedly high in satellite eNodeB even though there exists a single test UE with high CQI and MCS index values under satellite eNodeB. The number of RB utilization is expected to concentrate around high values in satellite eNodeB. However, DL PDCP throughput fluctuates in satellite eNodeB as better evidenced in Fig. 11b. The main reason for this phenomenon is that arriving GTP PDUs over satellite links are not regularly filling out the transmit buffer at satellite eNodeB. If the transmit buffer size becomes full, satellite eNodeB will be able to schedule and send PDCP PDUs appropriately to UEs in DL. However, this is not observed most of the time during our observation period. For this reason, distribution of number of $\mathrm{RB}$ utilization is more dispersed than expected. These unbalanced distributions in both FU ratio and number of RB utilization are mainly due to delay and jitter of satellite link.

The second observation states that in satellite link relatively low numbers of PDUs are generated at the RLC layer compared to the PDCP layer. The CQI values of the test UE for satellite are high, however high numbers of PDCP PDUs are also generated. The scheduler at the satellite eNodeB is concatenating PDCP PDUs and sending them to MAC layer as one Service Data Unit (SDU). However, this process of scheduler is delayed due to effect of satellite link. Under normal circumstances, schedulers are expected to segment PDCP PDUs at the RLC layer. However, this kind of segmentation does not appear in satellite eNodeB where RLC PDUs are segmented at the PDCP layer. For this reason, in case many UEs inside satellite eNodeB exist, the existence of high number of PDCP PDUs can create a transmit buffering problem.

The third observation states that the excessive existence of PDCP PDUs can be due to GTP-U accelerator. GTP-U accelerator technique includes a caching method. Therefore, we observe that there can be a trade-off between the number of UEs that can be supported within a eNodeB with satellitebased backhaul link and the caching size related to the GTP$\mathrm{U}$ accelerator's performance. Large caching size increases the capabilities of the GTP-U accelerator in terms of providing lower latency. On the other hand, it can also decrease the number of UEs that can be scheduled over the satellite eNodeB due to existence of large number of PDCP PDUs compared to RLC PDUs. Lower caching size on the other hand adversely effects the GTP-U's accelerator, but decreases the number of PDCP PDUs in the transmit buffer of the scheduler. Therefore, for higher performance gains in terms of low latency and high support of UEs for satellite eNodeB, an optimal balance between the caching size and the number of UEs using satellite eNodeB is needed. The reason is that GTP-U acceleration is a fundamental requirement for satellite links to decrease the inherent latency.

\section{CONCLUSIONS}

It is inevitable that satellite networks will integrate with other networks including 5G cellular networks. In this paper, we investigated various cellular network KPIs of both satellite and terrestrial eNodeBs in an experimental set-up to observe the effect of satellite-based backhaul links on radio network performance. The comparative experimental performance evaluation is performed in terms of CQI values, MCS index, PDCP throughput, number of utilized RBs, FU utilization ratios as well as BLER and MIMO TB utilization. Our experimental results indicate three main observations: The first one is the existence of RB and FU utilization problems in satellitebased backhaul network due to inherent delay and jitter on satellite links even though high MCS index and CQI values are monitored during observation intervals. The second one is that compared to terrestrial network total number of PDCP packets outnumbers the total number of RLC packets in satellitebased backhaul network. Our final observation designates that 
there will be a trade-off between the number of UEs that can be supported with satellite eNodeB and the caching size of the utilized GTP-U accelerator. Therefore, an optimal balance between the caching size of the GTP-U accelerator for latency reductions and the number of UEs using satellite eNodeB needs to be adjusted before deployment of satellite-based backhaul networks in an operational environment.

\section{ACKNOWLEDGMENTS}

This work was partially funded by Spanish MINECO grant TEC2017-88373-R (5G-REFINE) and by Generalitat de Catalunya grant 2017 SGR 1195.

\section{REFERENCES}

[1] M. Jia et al. Broadband hybrid satellite-terrestrial communication systems based on cognitive radio toward 5g. IEEE Wireless Communications, 23(6):96-106, December 2016.

[2] B. T. Jou et al. Architecture options for satellite integration into 5g networks. In 2018 European Conference on Networks and Communications (EuCNC), pages 398-9. IEEE, 2018.

[3] L. Boero et al. Satellite networking integration in the $5 \mathrm{~g}$ ecosystem: Research trends and open challenges. IEEE Network, 32(5):9-15, 2018.

[4] Calum Dewar and Dan Warren. Understanding 5g: Perspectives on future technological advancements in mobile. https://bit.ly/2PPli2M. [Online access; 23-April-2019].

[5] E. Zeydan et al. Big data caching for networking: Moving from cloud to edge. IEEE Communications Magazine, 54(9):36-42, 2016.

[6] Electronics Communication Committee (ECC). Satellite solutions for 5g. https://bit.ly/2shjhlm, 2018. [Online access; 23-April-2019].

[7] G. Giambene, S. Kota, and P. Pillai. Satellite-5g integration: A network perspective. IEEE Network, 32(5):25-31, 2018.

[8] M. Breiling et al. Lte backhauling over meo-satellites. In 2014 7th Advanced Satellite Multimedia Systems Conference and the 13th Signal Processing for Space Communications Workshop (ASMS/SPSC), pages 174-181. IEEE, 2014.

[9] A. Guidotti et al. Lte-based satellite communications in leo megaconstellations. International Journal of Satellite Communications and Networking, 2018.

[10] L. Ai. Ka-band HTS channel uplink SNIR probability model. International Journal of Satellite Communications and Networking, 36(1):146164, 2018.

[11] A. Kapovits et al. Satellite communications integration with terrestrial networks. China Communications, 15(8):22-38, 2018.

[12] A. Guidotti et al. Architectures and key technical challenges for $5 \mathrm{~g}$ systems incorporating satellites. IEEE Transactions on Vehicular Technology, 2019.

[13] F. Bastia et al. LTE adaptation for mobile broadband satellite networks. EURASIP Journal on Wireless Communications and Networking, 2009:12, 2009.

[14] N. Zangar and S. Hendaoui. Leveraging multiuser diversity for adaptive hybrid satellite-LTE downlink scheduler (H-MUDoS) in emerging 5Gsatellite network. International Journal of Satellite Communications and Networking, 2018

[15] G. Araniti et al. Multimedia content delivery for emerging 5G-satellite networks. IEEE Transactions on Broadcasting, 62(1):10-23, 2016.

[16] M. Shaat et al. Integrated terrestrial-satellite wireless backhauling: resource management and benefits for 5G. IEEE Vehicular Technology Magazine, 13(3):39-47, 2018.

[17] C. Niephaus et al. QoS Provisioning in Converged Satellite and Terrestrial Networks: A Survey of the State of-the-Art. IEEE Communications Surveys Tutorials, 18(4):2415-2441, 2016.

[18] multi-satellite relay transmission in 5g: Concepts, techniques, and challenges.

[19] R. Kumar et al. WiLiTV: Reducing Live Satellite TV Costs Using Wireless Relays. IEEE Journal on Selected Areas in Communications, 36(2):233-245, 2018

[20] 3GPP TR 38.811 V15.0.0. Study on New Radio (NR) to support non terrestrial networks (Release 15), (2018-06).

[21] ETSI. Work Programme (2017-2018). https://bit.ly/2H5a0Um, 2017. [Online; accessed 08-May-2019].

[22] ITU Satellite Symposium, 28 to 30 Nov. 2018, in Geneva, Switzerland.
[23] 5GPP. 5G Vision, The 5G Infrastructure PPP: the next generation of communication networks and services. https://bit.ly/1GZuqHW, 2018. [Online; accessed 12-October-2018].

[24] beam tracking for uav mounted satcom on-the-move with massive antenna array.

[25] M. Hasan and C. Bianchi. Ka band enabling technologies for high throughput satellite (hts) communications. International Journal of Satellite Communications and Networking, 34(4):483-501, 2016.

[26] Gilat Satellite Networks. GiLAT Capricorn Family. https://bit.ly/ 2DVwWom. [Online access; 30-January-2019].

[27] C. S. Modulo. GTP Acceleration Board. https://bit.ly/2JkK9ui. [Online access; 08-May-2019].

[28] S. Ahluwalia et al. Acceleration of GTP traffic flows, over a satellite link, in a terrestrial wireless mobile communications system, June 30 2016. US Patent App. 14/792,199.

[29] H. Fenech et al. High throughput satellite systems: An analytical approach. IEEE Transactions on Aerospace and Electronic Systems, 51(1):192-202, 2015.

[30] J. Dugan et al. iPerf3: The ultimate speed test tool for TCP, UDP and SCTP. https://iperf.fr/, 2018. [Online; accessed 11-October-2018].

[31] Infovista. TEMS Product Page. https://bit.ly/2PPzrx9, 2018. [Online; accessed 25-September-2018].

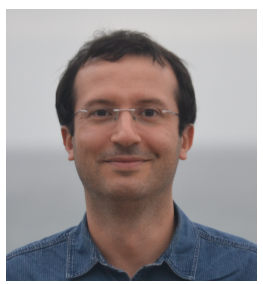

Engin Zeydan received his Ph.D. degree in February 2011 from the Department of Electrical and Computer Engineering at Stevens Institute of Technology, Hoboken, NJ, USA. Previously, he received his M.Sc. and B.Sc. degrees from the Department of Electrical and Electronics Engineering at Middle East Technical University, Ankara, Turkey, in 2006 and 2004, respectively. Dr. Zeydan has worked as an $R \& D$ engineer for Avea, a mobile operator in Turkey, between 2011 and 2016. He was with Turk Telekom Labs working as a Senior R\&D Engineer between 2016 and 2018. He was also part-time instructor at Electrical and Electronics Engineering department of Ozyegin University between 2015 to 2018. He is currently with Communication Networks Division of the Centre Tecnologic de Telecomunicacions de Catalunya (CTTC) working as a Researcher. He received Best Paper Award from the Network of Future Conference in 2017. His research interests are telecommunications and big data networking.

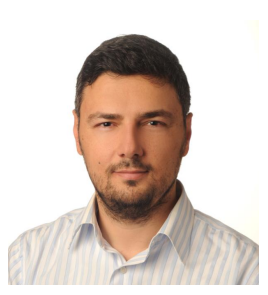

Yekta Turk received his Ph.D. degree in 2018 from the Department of Computer Engineering at Maltepe University, Istanbul, Turkey. Previously, he received his M.Sc. degree from in Telecommunications and Computer Networks from the George Washington University, DC, USA, in 2007 and B.Sc. degree in Electrics and Electronics Engineering from Anadolu University, Turkey, in 2005. He has worked for various fixed and mobile network operators more than 10 years. He is a mobile network architect based in Istanbul, Turkey. His research interests are mobile radio telecommunications and computer networks. 